\title{
Favorable effect of corticosteroids in treating acute-on- chronic liver failure underlying chronic hepatitis B
}

\author{
Hyeji Kim', Jung Hyun Kwon', Yong Hee Kim', Soon Woo Nam1', Jong Yul Lee', and Jeong Won Jang² \\ 'Department of Internal Medicine, The Catholic University of Korea Incheon St. Mary's Hospital, Incheon; 'Department of Internal \\ Medicine, The Catholic University of Korea Seoul St. Mary's Hospital, Seoul, Korea
}

Acute-on-chronic liver failure (ACLF) occurs in the presence of a chronic liver disease or cirrhosis, and often results from exacerbation of chronic hepatitis B (CHB). The efficacy of corticosteroid treatment in ACLF patients with underlying CHB remains unclear. We report the case of a 50-year-old woman who experienced ACLF due to CHB exacerbation and was treated with a combination of corticosteroids and nucleot(s)ide analogue (NUC). The patient showed rapid decompensation due to CHB exacerbation. Three months of antiviral therapy produced no improvement in liver function. Combination therapy with corticosteroids and NUC was started, which did result in improvement of liver function. This case shows that the combined therapy of corticosteroids and NUC can be effective in treating ACLF due to CHB exacerbation. (Clin Mol Hepatol 2018;24:430-435)

Keywords: Acute-on-chronic liver failure; Chronic hepatitis B; Corticosteroid; Exacerbation; Reactivation

\section{INTRODUCTION}

Acute-on-chronic liver failure (ACLF) has emerged as a distinct clinical entity and manifests as liver failure in the presence of chronic liver disease or cirrhosis. An acute insult such as exacerbation of chronic hepatitis B (CHB), alcohol consumption, drug-induced liver injury and other viral hepatitis can lead to ACLF.' The pathophysiology of ACLF relates to persistent inflammation, immune dysregulation with a systemic inflammatory response syndrome and subsequent sepsis due to immune paresis.'

Acute exacerbation of CHB can lead to fatal liver failure characterized by a high alanine aminotransferase level, jaundice, and coagulopathy. ${ }^{2}$ The use of nucleot(s)ide analogues (NUCs) can be effective in managing CHB exacerbation, but their efficacy in pre- venting rapid progression to ACLF is limited. ${ }^{3}$ The main pathogenesis of CHB exacerbation is considered to be liver injury through the induction of cytotoxic T-lymphocyte-mediated cytolytic pathways in hepatitis B virus (HBV)-infected hepatocytes. ${ }^{4}$ Combined administration of corticosteroids with NUCs may counter liver function deterioration in patients with ACLF and can inhibit the immune-mediated reaction of T-lymphocyte, thus preventing cytolysis of infected hepatocytes. ${ }^{5}$ However, in some cases, the corticosteroid treatment may enhance replication of HBV DNA, aggravating the condition, and lead to fulminant hepatic failure. Therefore, the efficacy of corticosteroid in managing acute exacerbation of $\mathrm{CHB}$ is unclear.

Here, we report a case of successful treatment of ACLF with underlying CHB with combined with corticosteroids and NUC therapy.

\footnotetext{
Abbreviations:

ACLF, acute-on-chronic liver failure; $\mathrm{AlH}$, autoimmune hepatitis; $\mathrm{ANA}$, antinuclear antibody; CHB, chronic hepatitis B; CT, computed tomography; HAART, highly active antiretroviral therapy; HBV, hepatitis B virus; HCV, hepatitis $C$ virus; HIV, human immunodeficiency virus; IgM, immunoglobulin M; MELD, model for end stage liver disease; NUC, nucleot(s)ide analogue; RTE, real-time elastography; PT, prothrombin time
}

\section{Corresponding author : Jung Hyun Kwon}

Department of Internal Medicine, The Catholic University of Korea Incheon St. Mary's Hospital, 56 Dongsu-ro, Bupyeong-gu, Incheon 21431, Korea Tel: +82-32-280-5020, Fax: +82-32-280-5987

E-mail: doctorkwon@catholic.ac.kr

https://orcid.org/0000-0002-5484-5864 


\section{CASE REPORT}

A 50-year-old woman was referred to our hospital for liver transplantation. She was diagnosed with CHB approximately 20 years ago, but was not receiving any antiviral treatment. Before being referred to our hospital, she was admitted to another hospital due to CHB flare up (Fig. 1A). She denied alcohol consumption and taking no other medications or herbal supplements. She was treated with tenofovir 300 mg daily. However, despite onemonth treatment with tenofovir, newly developed ascites were seen and jaundice progressed; the total bilirubin level elevated 4 times higher than initial value. After that, she kept treatment with medication and underwent intermittent abdominal paracentesis for 2 months. Because there was no improvement of liver function, she referred to our hospital. At the time of admission to our hospital, she had a blood pressure of $100 / 70 \mathrm{mmHg}$; pulse rate, 103 beats/min; respiration rate, 20 breaths/min; and temperature, $36.6^{\circ} \mathrm{C}$, with a clear consciousness. Physical examination showed icteric sclerae, a distended abdomen without tenderness, and pitting edema. Results of laboratory tests on admission were as fol- lows: albumin level, 2.4g/dL (3.8-5.1 g/dL); total bilirubin level, $15.4 \mathrm{mg} / \mathrm{dL}$ (0.2-1.4 mg/dL); direct bilirubin level, $7.9 \mathrm{mg} / \mathrm{dL}$ (0-0.3 $\mathrm{mg} / \mathrm{dL}) ;$ alkaline phosphatase level, $196 \mathrm{IU} / \mathrm{L}$ (38-110 IU/L); gamma-glutamyl transpeptidase level, $36 \mathrm{IU} / \mathrm{L}$ (0-38 IU/L); aspartate aminotransferase level, $113 \mathrm{IU} / \mathrm{L}$ (9-40 IU/L); alanine aminotransferase level, $61 \mathrm{IU} / \mathrm{L}$ (9-40 IU/L); hemoglobin level, $12.1 \mathrm{~g} / \mathrm{dL}$ (12$15 \mathrm{~g} / \mathrm{dL})$; white blood cell count, $8.94 \times 10^{3} / \mu \mathrm{L}\left(4-9.9 \times 10^{3} / \mu \mathrm{L}\right)$; platelet count, $96 \times 10^{3} / \mu \mathrm{L}\left(140-400 \times 10^{3} / \mu \mathrm{L}\right)$; creatinine level, 0.9 $\mathrm{mg} / \mathrm{dL}$ (0.5-1.2 mg/dL); and prothrombin time (PT), 17.7 seconds (10.4-12.9 seconds), with a calculated Child-Pugh score of 11 (C) and a model for end-stage liver disease (MELD) score of 20. $\mathrm{HBeAg}$ test result was positive, while anti-HBe test result was negative. HBV DNA level was already less than $20 \mathrm{IU} / \mathrm{mL}$ (Table 1). Serologic tests for immunoglobulin M (IgM) anti-hepatitis A virus, anti-hepatitis C virus (HCV) antibody, HCV real-time polymerase chain reaction, human immunodeficiency virus (HIV) antigen, antiHIV antibody, IgM anti-cytomegalovirus, IgM anti-Epstein-Barr virus viral-capsid antigen were negative. C-reactive protein level was $5.55 \mathrm{mg} / \mathrm{L}$ (0-5 mg/L). Ascites study revealed that serum-ascites albumin gradient was $2.2 \mathrm{~g} / \mathrm{dL}$ and absolute neutrophil
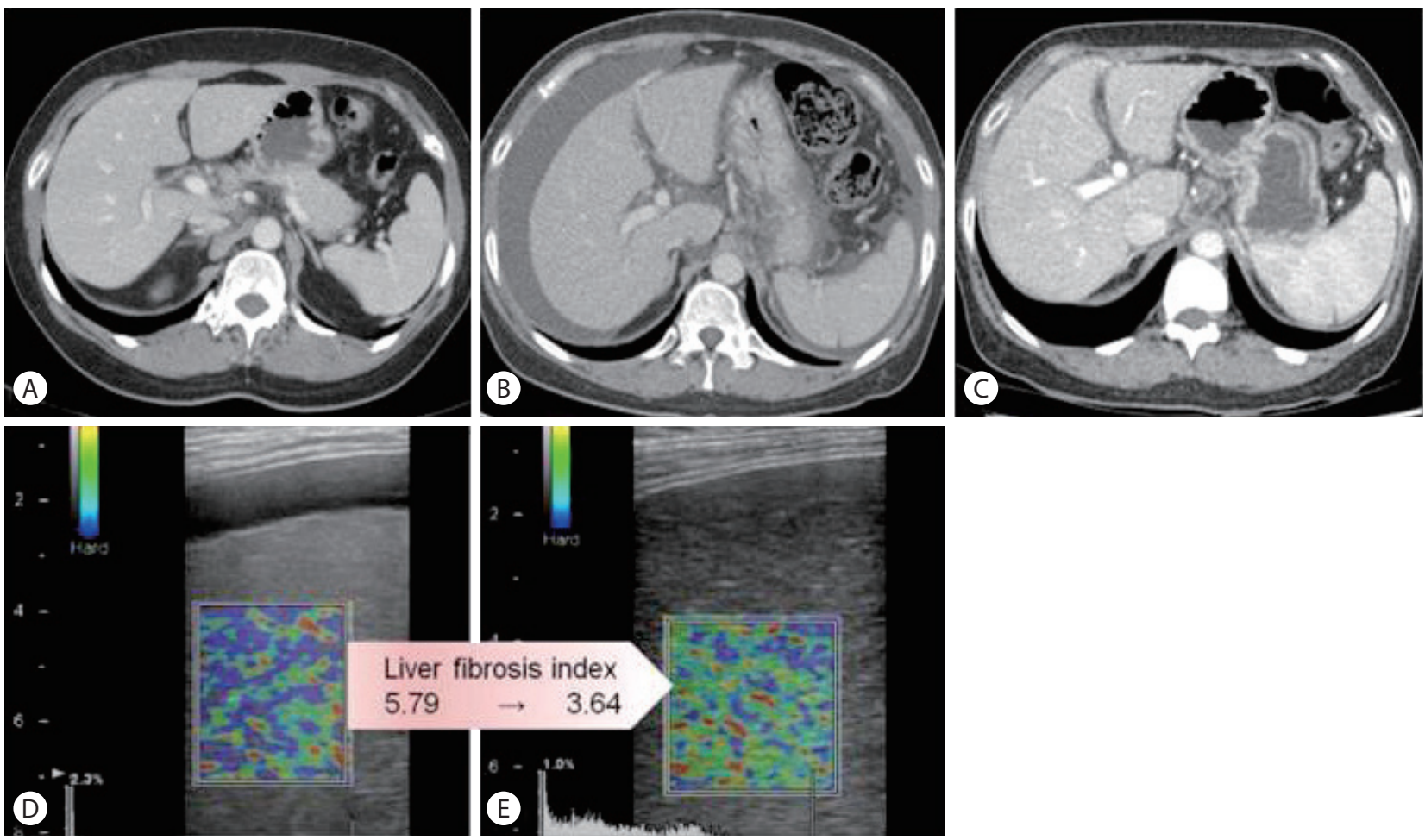

Figure 1. Dynamic contrast-enhanced computed tomography (CT) images in the portal venous phase $(A-C)$ and real-time elastography (RTE) images (D, E). (A) CT scan obtained 3 months before admission to our hospital showing a cirrhotic liver. (B) Upon admission to our hospital, a moderate amount of ascites was observed due to acute-on-chronic liver failure (ACLF) with chronic hepatitis B (CHB). (C) CT scan showing that combined treatment with a corticosteroid and a nucleot(s)ide analogue (NUC) decreased the amount of ascites. (D, E) RTE images obtained after combined treatment show an improved liver fibrosis index. 
Table 1. Serial levels of laboratory parameters before and after combined treatment with a nucleot(s)ide analogue and a corticosteroid

\begin{tabular}{lccccc}
\hline & $\begin{array}{c}\text { Tenofovir start } \\
\text { (initial visit to } \\
\text { another hospital) }\end{array}$ & Tenofovir 1 month & $\begin{array}{c}\text { Tenofovir 3 months } \\
\text { (referral to our } \\
\text { hospital) }\end{array}$ & $\begin{array}{c}\text { Tenofovir 4 months } \\
\text { (corticosteroid } \\
\text { treatment 3 weeks) }\end{array}$ & Tenofovir 12 months \\
\hline TB (mg/dL) & 3.6 & 16.06 & 15.4 & 4.8 & 1.3 \\
\hline ALT (IU/L) & 1,102 & 34 & 61 & 34 & 18 \\
Albumin (g/dL) & 3.9 & 2.7 & 2.4 & 2.7 & 4.2 \\
PT (INR) & 1.17 & 1.52 & 1.49 & 1.28 & 1.11 \\
\hline
\end{tabular}

TB, total bilirubin; ALT, alanine aminotransferase; PT, prothrombin time; INR, international normalized ratio.

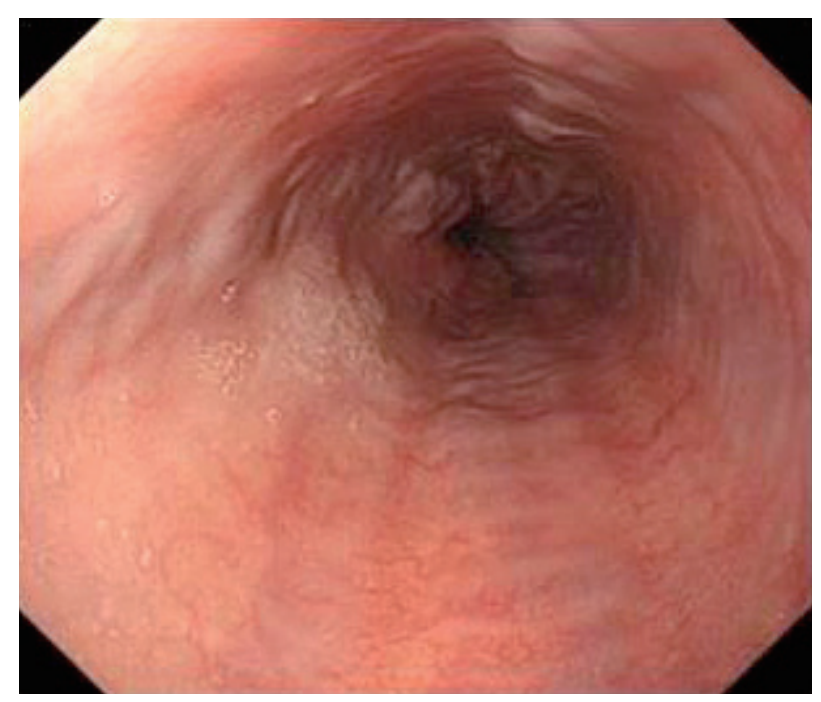

Figure 2. Esophagogastroduodenoscopy image showing grade 1 varices in the lower esophagus.

count was 10 cells/ $\mu \mathrm{L}$. Dynamic contrast-enhanced computed tomography (CT) showed a cirrhotic configuration of the liver with mild splenomegaly and moderate amount of ascites (Fig. 1B). Esophagogastroduodenoscopy revealed grade 1 esophageal varix (Fig. 2). While waiting for a cadaveric liver donor, she underwent abdominal paracentesis once or twice a week. The total bilirubin level and PT did not improve but remained unchanged. Her performance status remained at grade 1 .

To rule out other concomitant causes of liver function deterioration, further evaluation for autoimmunity was performed. The anti-nuclear antibody (ANA) test result was weakly positive, at a titer of 1:80. The anti-mitochondrial antibody, anti-smooth muscle antibody and anti-liver kidney microsome antibody-1 test results were negative, and immunoglobulin $\mathrm{G}$ level was $1,338.1 \mathrm{mg} / \mathrm{dL}$ (800-1,800 mg/dL). Although we considered percutaneous liver biopsy, we couldn't do it because bleeding risk was higher due to PT prolongation and moderate amount of ascites.

Because there was no improvement in liver function despite treatment with NUC for 3 months and liver donor was unavailable, prednisolone was started after careful consideration. Although the patient did not show a definite evidence of autoimmunity, we speculated that she had excessive immune activity due to ACLF with CHB. After confirming that HBV DNA was undetectable and chest radiography and urinalysis showed no evidence of infection, prednisolone therapy was started at $30 \mathrm{mg} /$ day.

Shortly after combination therapy with corticosteroid and NUC, resolution of ACLF was seen. Total bilirubin level decreased by 2 to $5 \mathrm{mg} / \mathrm{dL}$ every 7 days, decreasing from $15.4 \mathrm{mg} / \mathrm{dL}$ to $5.6 \mathrm{mg} / \mathrm{dL}$ in only 3 weeks (Fig. 3). Prednisolone dose was tapered by 5 or $10 \mathrm{mg}$ at least every 4 days, to $5 \mathrm{mg}$, depending on the decreasing trend of the total bilirubin level. Prednisolone therapy was subsequently stopped. The patient was discharged on tenofovir and oral diuretics 1 week after the cessation of combination therapy. Abdominal paracentesis was not needed after discharge. On oneyear follow-up, her Child-Pugh score and MELD score improved to 5(A) and 4, respectively. The amount of ascites decreased on follow-up CT (Fig. 1C). Her elastography scores for liver fibrosis also improved from 5.79 to 3.64 (Fig. 1D, E). During the follow-up period, the ANA converted negative on 6 months after admission.

\section{DISCUSSION}

Up to $30 \%$ of patients with CHB experience spontaneous reactivation of hepatitis every year, and up to $8 \%$ of patients develop decompensation, that is, severe acute exacerbation, and may progress to ACLF. ${ }^{1,6}$ Reactivation of HBV could be due to changes in the immunological control of viral replication and reconstitution of host defenses.' The prognosis of patients with severe acute exacerbation of CHB leading to ACLF is extremely poor. ${ }^{2}$

In acute exacerbation of $\mathrm{CHB}$, administration of NUCs results in rapid cessation of HBV DNA replication and thus reduced HBV DNA level. We used tenofovir as the NUC in this case. A random- 


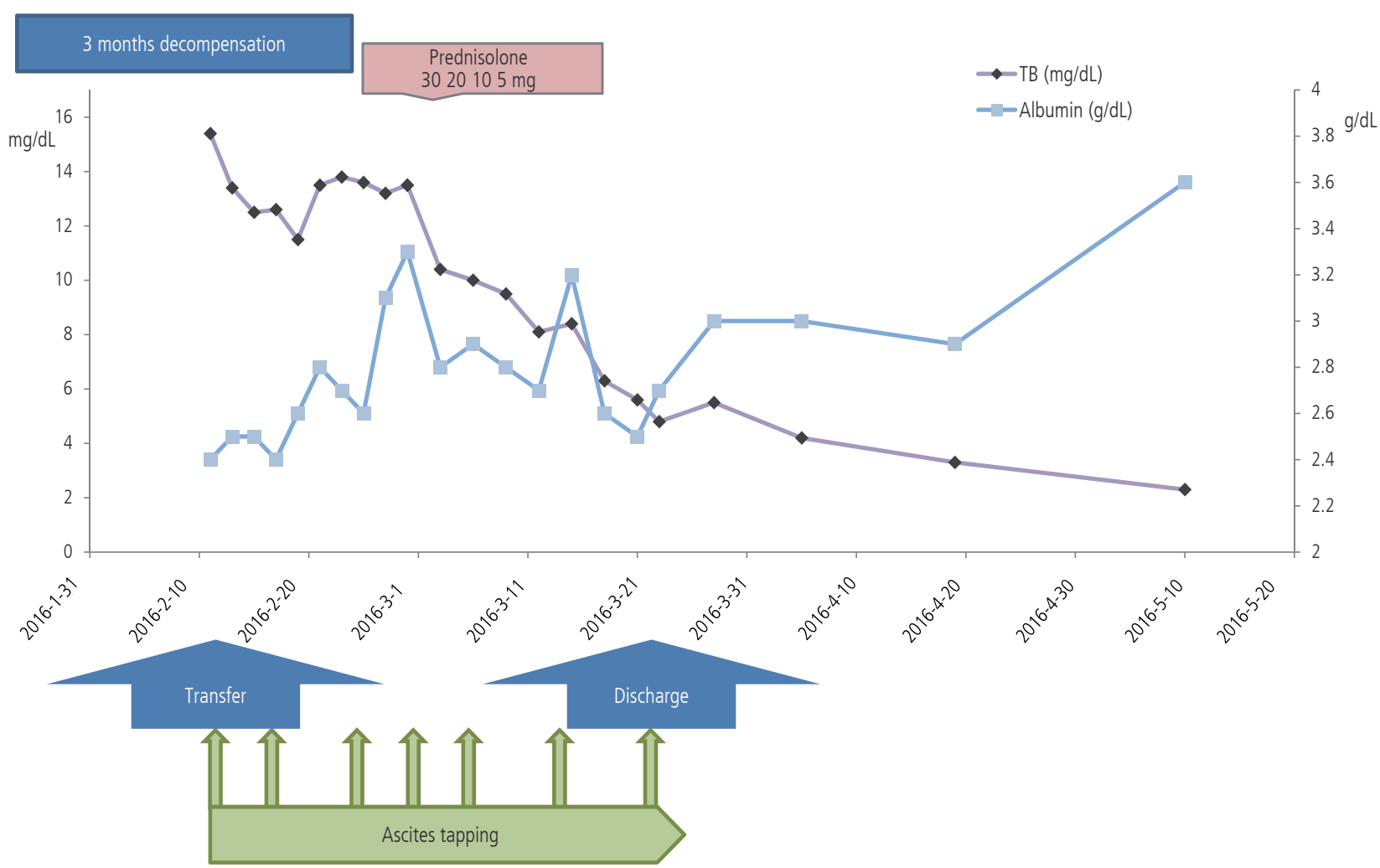

Figure 3. Clinical course of the patient with ACLF with CHB showing a favorable effect of combined corticosteroid and NUC treatment. TB, total bilirubin; ACLF, acute-on-chronic liver failure; CHB, chronic hepatitis B; NUC, nucleot(s)ide analogue.

ized controlled trial showed that reducing the HBV DNA level by $>2$ logs from baseline within 2 weeks using tenofovir improved the transplant-free survival rate. ${ }^{7}$ However, the improvement in liver function with administration of NUCs may take a few weeks to a few months, during which period excessive immune activity may continue and liver cell injury may progress. ${ }^{8}$ The corticosteroid can modulate the activity of CHB during this period by suppressing the host immune response to HBV antigens. ${ }^{5}$ Therefore, administering corticosteroids to patients with ACLF with underlying CHB is a reasonable treatment decision. ${ }^{9}$ A recent meta-analysis showed that treatment with corticosteroids can significantly increase the survival rate of patients with severe hepatitis B infection. ${ }^{10}$ This study showed that the corticosteroid treatment is more effective in patients at the pre-liver failure stage and is not associated with the development of secondary infection and bleeding.

However, reactivation of the HBV in patients with malignant, inflammatory and autoimmune conditions who undergo immunosuppression has been widely reported. Higher HBV reactivation rates have been observed in patients with hematological malignancies receiving treatment regimens that utilize high-dose corticosteroids and/or rituximab. ${ }^{11,12}$ Patients who are HBsAg negative/ hepatitis B core antibody positive also remain at risk of HBV reactivation in the setting of immunosuppression owing to the persistence of the HBV in the form of cccDNA in hepatocytes and other tissues, ${ }^{13}$ and long-term use of corticosteroids particularly increases the risk of HBV reactivation. ${ }^{10}$ Therefore, administration of NUCs for HBV replication inhibition combined with a short-term corticosteroid therapy should be considered in such risky patients. $^{3,8,14}$

Several studies have reported on the importance of early introduction of corticosteroid treatment in ACLF with $\mathrm{CHB}_{1}^{3,8,9}$ as delayed introduction, for example, after 10 days, may not be effective because a large number of hepatocytes might have already been destroyed and inhibition of the inflammatory reaction might not be effective. ${ }^{3,8}$ However, "early stage" or "pre-liver failure" in CHB exacerbation has not yet been clearly defined. In this case, corticosteroid therapy was administered after 3 months of NUC treatment and maintained for 3 weeks.

Our patient maintained a good performance status and liver function without further deterioration while waiting for the liver donor. This indicated that not all hepatocytes of the patient were destroyed. Furthermore, the ANA test showed weak positivity; 
false positivity in sometimes seen in cases of CHB exacerbation. We suspected combined autoimmunity in our patient. Therefore, a moderate corticosteroid dose was used, unlike in reported studies, whereby an initial dose of $40 \mathrm{mg}$ or more daily prednisolone was administered without evidence of autoimmune hepatitis (AlH). ${ }^{8-10,15,16}$ There have been several case reports of AlH developed as part of immune reconstitution inflammatory syndrome after highly active antiretroviral therapy (HAART) in HIV infected patients. ${ }^{17}$ The mechanism that results in AIH after initiation of HAART in HIV patient is probably involves changes in CD4+ T cells. ${ }^{18}$ The rapid increase in CD4+ T cells may produce a larger proportion of Th17 cells that release IL-17 without appropriate Treg cells numerical and functional reconstitution, which are similar to AlH. Infiltration of CD 4+ T cells in the liver are increased in patients with $\mathrm{AlH}$ and IL-17 signaling pathway play a critical role in the pathogenesis of AlH. Likewise, it is possible to hypothesize that immune dysregulation caused by ACLF was modulated after NUC treatment and subsequent autoimmunity was induced by restored immunity. It suggests that immune reconstitution after NUC treatment had a role for good response to corticosteroid treatment although the patient was treated with delayed introduction after the diagnosis of $\mathrm{CHB}$ exacerbation.

In our patient, no newly developed infection, bleeding, or HBV reactivation was seen during corticosteroid treatment. HBV DNA suppression was enough adequate and the possibility of infection was ruled out before initiating the corticosteroid treatment. Adverse effects of corticosteroid combination therapy such as pneumonia or enteritis have been reported, ${ }^{2}$ but there are no reports on $\mathrm{HBV}$ reactivation.

In conclusion, this case suggests that combined corticosteroid and NUC therapy may be effective in patients with ACLF with $\mathrm{CHB}$, particularly in those with suspected autoimmunity. However, a careful consideration of patient condition prior to initiation and monitoring for infection are necessary.

\section{Authors' contribution}

Jung Hyun Kwon planed the study and reviewed the references. Hyeji Kim and Jung Hyun Kwon wrote the paper. Yong Hee Kim, Soon Woo Nam and Jong Yul Lee treated and reviewed the patient. Jeong Won Jang gave a comment for consultation.

\section{Conflicts of Interest}

The authors have no conflicts to disclose.

\section{REFERENCES}

1. Sarin SK, Choudhury A. Acute-on-chronic liver failure: terminology, mechanisms and management. Nat Rev Gastroenterol Hepatol 2016;13:131-149.

2. Yasui S, Fujiwara K, Nakamura M, Miyamura T, Yonemitsu Y, Mikata $R$, et al. Virological efficacy of combination therapy with corticosteroid and nucleoside analogue for severe acute exacerbation of chronic hepatitis B. J Viral Hepat 2015;22:94-102.

3. Fujiwara K, Yasui S, Okitsu K, Yonemitsu Y, Oda S, Yokosuka O. The requirement for a sufficient period of corticosteroid treatment in combination with nucleoside analogue for severe acute exacerbation of chronic hepatitis B. J Gastroenterol 2010;45:1255-1262.

4. Chisari FV, Ferrari C. Hepatitis B virus immunopathogenesis. Annu Rev Immunol 1995;13:29-60.

5. Sjogren MH, Hoofnagle JH, Waggoner JG. Effect of corticosteroid therapy on levels of antibody to hepatitis $B$ core antigen in patients with chronic type B hepatitis. Hepatology 1987;7:582-585.

6. Levy P, Marcellin P, Martinot-Peignoux M, Degott C, Nataf J, Benhamou JP. Clinical course of spontaneous reactivation of hepatitis $B$ virus infection in patients with chronic hepatitis B. Hepatology 1990;12:570-574.

7. Garg H, Sarin SK, Kumar M, Garg V, Sharma BC, Kumar A. Tenofovir improves the outcome in patients with spontaneous reactivation of hepatitis B presenting as acute-on-chronic liver failure. Hepatology 2011;53:774-780

8. Fujiwara K, Yasui S, Yonemitsu Y, Fukai K, Arai M, Imazeki F, et al. Efficacy of combination therapy of antiviral and immunosuppressive drugs for the treatment of severe acute exacerbation of chronic hepatitis B. J Gastroenterol 2008;43:711-719.

9. Zhang $X Q$, Jiang L, You JP, Liu YY, Peng J, Zhang HY, et al. Efficacy of short-term dexamethasone therapy in acute-on-chronic pre-liver failure. Hepatol Res 2011;41:46-53.

10. He B, Zhang Y, Lü MH, Cao YL, Fan YH, Deng JQ, et al. Glucocorticoids can increase the survival rate of patients with severe viral hepatitis B: a meta-analysis. Eur J Gastroenterol Hepatol 2013;25:926934.

11. Cheng AL, Hsiung CA, Su IJ, Chen PJ, Chang MC, Tsao CJ, et al. Steroid-free chemotherapy decreases risk of hepatitis B virus (HBV) reactivation in HBV-carriers with lymphoma. Hepatology 2003;37:1320-1328.

12. Wu CY, Hsiao LT, Chiou TJ, Gau JP, Liu JH, Yu YB, et al. Lymphocyte/ monocyte ratio and cycles of rituximab-containing therapy are risk factors for hepatitis B virus reactivation in patients with diffuse large B-cell lymphoma and resolved hepatitis B. Leuk Lymphoma 2015;56:2357-2364.

13. Chemin I, Jeantet D, Kay A, Trépo C. Role of silent hepatitis B virus in chronic hepatitis B surface antigen(-) liver disease. Antiviral Res 
Hyeji Kim, et al.

Corticosteroid in acute-on-chronic liver failure with hepatitis B

2001;52:117-123.

14. Wong VW, Wong GL, Yiu KK, Chim AM, Chu SH, Chan HY, et al. Entecavir treatment in patients with severe acute exacerbation of chronic hepatitis B. J Hepatol 2011;54:236-242.

15. Ware A, Cuthbert J, Shorey J, Gurian L, Eigenbrodt E, Combes B. A prospective trial of steroid therapy in severe viral hepatitis. The prognostic significance of bridging necrosis. Gastroenterology 1981;80:219-224.

16. Gregory PB, Knauer CM, Kempson RL, Miller R. Steroid therapy in severe viral hepatitis. A double-blind, randomized trial of methylprednisolone versus placebo. N Engl J Med 1976;294:681-687.

17. O'Leary JG, Zachary K, Misdraji J, Chung RT. De novo autoimmune hepatitis during immune reconstitution in an HIV-infected patient receiving highly active antiretroviral therapy. Clin Infect Dis 2008;46:e12-e14.

18. Murunga E, Andersson M, Rensburg CV. Autoimmune hepatitis: a manifestation of immune reconstitution inflammatory syndrome in HIV infected patients? Scand J Gastroenterol 2016;51:814-818. 